\title{
AC 2012-4831: UNDERSTANDING THE DIFFERENCE BETWEEN CLASS- ROOM LEARNING AND ONLINE LEARNING ON MEDICAL IMAGING WITH COMPUTER LAB EXERCISES
}

\section{Prof. Hong Man, Stevens Institute of Technology}

Hong Man joined the faculty of electrical and computer engineering at Stevens in Jan. 2000. He received his Ph.D. degree in electrical engineering from the Georgia Institute of Technology in Dec. 1999. Man is currently an Associate Professor in the Department of ECE. He is serving as the Director of the undergraduate Computer Engineering program, and the Director of the Visual Information Environment Laboratory at Stevens. His research interests have been in image and video processing, medical imaging, data analysis and pattern recognition. He has authored or co-authored more than 60 technical journal and conference papers on these topics. He is a Senior Member of IEEE and member of ASEE. 


\title{
Understanding the Difference between Classroom Learning and Online Learning on Medical Imaging with Computer Lab Exercises
}

\begin{abstract}
In this paper we present a study on the effectiveness of using a computer simulation software, SimuRad, in an undergraduate Medical Imaging course. This course is offered regularly in two different modes, i.e. an on-campus section in every Fall semester and an on-line section in every Spring semester. This enables us to compare students' learning experience with the same software in different environment. In this study, we focus on statistical analysis of three derived factors from our assessment results, i.e. performance improvement, difficulty level and usability satisfaction. We intend to identify the statistical relationships among these factors under classroom learning and online learning modes. Through this study, we are able to verify some hypotheses we introduced in our previous works. In particular, we will show that there can be no significant deficiency for online students to understand course content with the help of the computer lab exercises, and in certain scenarios, computer labs are more helpful to online students. The development and assessment of this software is partially supported by an NSF CCLI grant.
\end{abstract}

\section{Introduction}

A comprehensive "medical imaging" course in a bio-medical or bio-engineering curriculum may cover fundamental science and engineering principles (e.g. atomic and nuclear physics, Fourier analysis and reconstruction, and computer assisted tomography), medical imaging modalities (e.g. X-ray radiography, x-ray CT, nuclear medicine gamma imaging, magnetic resonance imaging, and ultrasound imaging), and clinical imaging practices (e.g. image analysis, visualization, instrumentation, and radiological protection $)^{1,2}$. Such a course has also been offered as an elective course in many graduate engineering and science programs ${ }^{3}$.

In order to offer this as an introductory undergraduate course, it is necessary to emphasize conceptual learning through lab exercises ${ }^{4,5}$. We have designed a series of computer lab exercises based on a newly developed computer simulation software tool - SimuRad ${ }^{6}$, which implements numerical algorithms to simulate the physical and biological processes in many common medical imaging modalities. The software contains expandable modules, each to support a serious lab exercises related to a particular modality. Currently implemented modules include math fundamentals, computed tomography (CT), x-ray physics, nuclear magnetic resonance (NMR), image enhancement and analysis. With these modules, seven computer lab exercises have been designed.

Lab 1, Convolution and Fourier Transform (math preparation)

Lab 2, Projection and Projection Slice Theorem (tomography)

Lab 3, Frequency domain reconstruction - interpolation methods (x-ray CT, MRI)

Lab 4, Filtered back projection - filtering, noise effects (x-ray CT)

Lab 5, X-ray attenuation coefficient and survival probability (x-ray)

Lab 6, NMR signals - precessions, relaxation, basic sequences (MRI)

Lab 7, Brain activation detection in fMRI (image analysis) 
In the undergraduate Bio-Medical Engineering (BME) program at Stevens Institute of Technology, "Medical Imaging" is offered each year in the Fall semester as a regular on-campus course, and in the Spring semester as an on-line course. In the on campus sections, the course constitutes a1.5-hour lecture and a 2-hour lab exercises. In the online sections, students are advised to spend the same amount of time for reading lecture notes and taking lab exercises. Students typically took 9 to 10 weeks to complete all seven labs, as described in the following sections. Upon completing each lab exercise, students are required to write a lab report. The contents of the lab exercises, e.g. procedures and results, were included in the midterm and final exams. We typically had around 30 students enrolled in the Fall semester and around 20 students in the Spring semester. The students are from the same student group each year, and mostly of them are our on-campus undergraduate BME students. The reason they took the course in different semester was mostly because of scheduling issues.

We started the deployment and assessment of these lab exercises in Fall 2008, and by Fall 2011 we have obtained the assessment results for three consecutive years. These results enable us to study student learning behaviors and performance in many different ways. In particular, we attempt to have a comparative study on students' learning experience in these computer-based lab exercises during the regular on campus sections (F08, F09 and F10) and during asynchronous online sections (S09, S10 and S11).

\section{Assessment Methodology}

Our assessment of students' learning experience is mainly based on student surveys. We designed a simple set of survey questions for students to complete after each lab exercise. The survey was voluntary. The questions include the scales of student's understand of a certain concept before and after the lab exercise, the scale of knowledge preparation for the lab exercise, the time spent on the lab exercise, and the need for lab design improvement. Following is an example of survey instruction provided in a lab assignment.

Answer the following survey questions using the scale $1 \sim 5$ (1: strongly disagree, 5: strongly agree):

1. You understand the concept of "filtered back projection method" BEFORE you take this lab exercise. 122345

2. You understand the concept of "filtered back projection method" AFTER you take this lab exercise. 122345

3. You have the knowledge and skill to complete this lab exercise without additional study beyond the lectures. $\begin{array}{lllll}1 & 2 & 3 & 4 & 5\end{array}$

4. This lab exercise takes you too much time. $\begin{array}{lllll}1 & 2 & 3 & 4 & 5\end{array}$

5. You think a better lab exercise can be designed to reach the objectives of this lab exercise. 122345

We have the following considerations in the design of the survey questions: 
- The difference between the Q1 score and the Q2 score roughly represents the student's perceived performance improvement.

- The Q4 score, normalized by the Q3 score, roughly indicates the difficulty level of a particular lab.

- The Q5 score indicates the need to improve the usability of the lab.

Accordingly we extract three metrics for assessment purposes:

- $\quad$ Learning improvement index $(\mathrm{LII})=(\mathrm{Q} 2$ _score - Q1_score $) / \mathrm{Q} 1$ score,

- $\quad$ Normalized difficulty index (NDI) $=$ Q4_score / Q3_score,

- Usability satisfaction index $(\mathrm{USI})=(6-\mathrm{Q} 5) / 5$.

To quantitatively analyze the assessment metrics, we introduce a set of hypothesis tests to evaluate the statistical significance of these assessment metrics between on-campus learning and on-line learning.

In the hypothesis testing, we calculate the mean and the standard deviation using the following equations,

$$
\begin{aligned}
& \mu=\frac{1}{N} \sum_{i=1}^{N} x_{i}, \\
& \sigma=\sqrt{\frac{N \sum_{i=1}^{N} x_{i}^{2}-\left(\sum_{i=1}^{N} x_{i}\right)^{2}}{N(N-1)}}
\end{aligned}
$$

where $\boldsymbol{x}_{\mathbf{i}}$ is certain assessment metric score, and $\boldsymbol{N}$ is the number of received survey.

Then we formulate three hypotheses as:

1. $\boldsymbol{H}_{10}: \boldsymbol{\mu}_{1}=\boldsymbol{\mu}_{2} \quad$ vS $\quad \boldsymbol{H}_{11}: \boldsymbol{\mu}_{1} \neq \boldsymbol{\mu}_{2}$

2. $\boldsymbol{H}_{20}: \boldsymbol{\mu}_{1}<\boldsymbol{\mu}_{2} \quad$ vS $\quad \boldsymbol{H}_{21}: \boldsymbol{\mu}_{1} \geq \boldsymbol{\mu}_{2}$

3. $\boldsymbol{H}_{30}: \boldsymbol{\mu}_{1}>\boldsymbol{\mu}_{2}$ vs $\boldsymbol{H}_{31}: \boldsymbol{\mu}_{1} \leq \boldsymbol{\mu}_{2}$

where $\boldsymbol{\mu}_{1}$ denotes the mean value of certain assessment metric from the on-campus section, and $\boldsymbol{\mu}_{2}$ denotes the mean value of the assessment metric from the corresponding online section in the same school year.

The test statistic is calculated as follows:

$$
t=\frac{\mu_{1}-\mu_{2}}{\sqrt{\frac{\sigma_{1}^{2}}{N_{1}}+\frac{\sigma_{2}^{2}}{N_{2}}}}
$$


We conduct the pairwise hypothesis testing between the mean value $\boldsymbol{\mu}_{1}$ of the on-campus sections and the mean value $\boldsymbol{\mu}_{2}$ of those on-line sections in each school year. Limiting the comparative study within each school year is intended to compensate the variations in student preparation levels over the years.

For each hypothesis testing, we will accept $\boldsymbol{H}_{10}$ if $|\boldsymbol{t}|<\mathbf{2 . 3 4 5}$, i.e., two set of assessment results are statistically equivalent (where 2.345 is for a two-tailed test where the results are significant with $\boldsymbol{p}=0.02$ and a one-tailed test where the results are significant with $\boldsymbol{p}=0.01$ ). We will accept $\boldsymbol{H}_{20}$ if $\boldsymbol{t}<\mathbf{- 2 . 3 4 5}$, i.e., the particular assessment index is more significant in on-line section; and we will accept $\boldsymbol{H}_{30}$ if $\boldsymbol{t}>\mathbf{2 . 3 4 5}$, i.e., this assessment index is more significant in oncampus section.

\section{Descriptions of the Lab Exercises and Assessment Results}

We have collected the survey results from the Fall 2008, Spring 2009, Fall 2009, Spring 2010, Fall 2010 and Spring 2011 semesters. The response rates varied from 50\% to $75 \%$. The overall average survey scores for each lab and in each semester are shown in Figures 1 7. The results are grouped into on-campus sections (F08, F09 and F10) and on-line sections (S09, S10 and S11) for each lab. The statistical test results of the three derived assessment metrics are presented in Tables 1 7.

\section{Lab 1. Convolution and Fourier Transform (math preparation)}

Student generates different signals by selecting multiple simple waveforms, e.g. sine, square. The amplitude, frequency and phase of each simple waveform are specified by the student. Then Fourier Transform is performed and the frequency response is displayed for each generated signal. Student is instructed to try a sequence of parameter sets to observe the changes of frequency responses corresponding to changes in signals. Student then selects a filter. Convolution of a signal with the filter is implemented through multiplication in frequency domain, which is to demonstration the concept that filtering is a process of frequency selective attenuation or amplification.
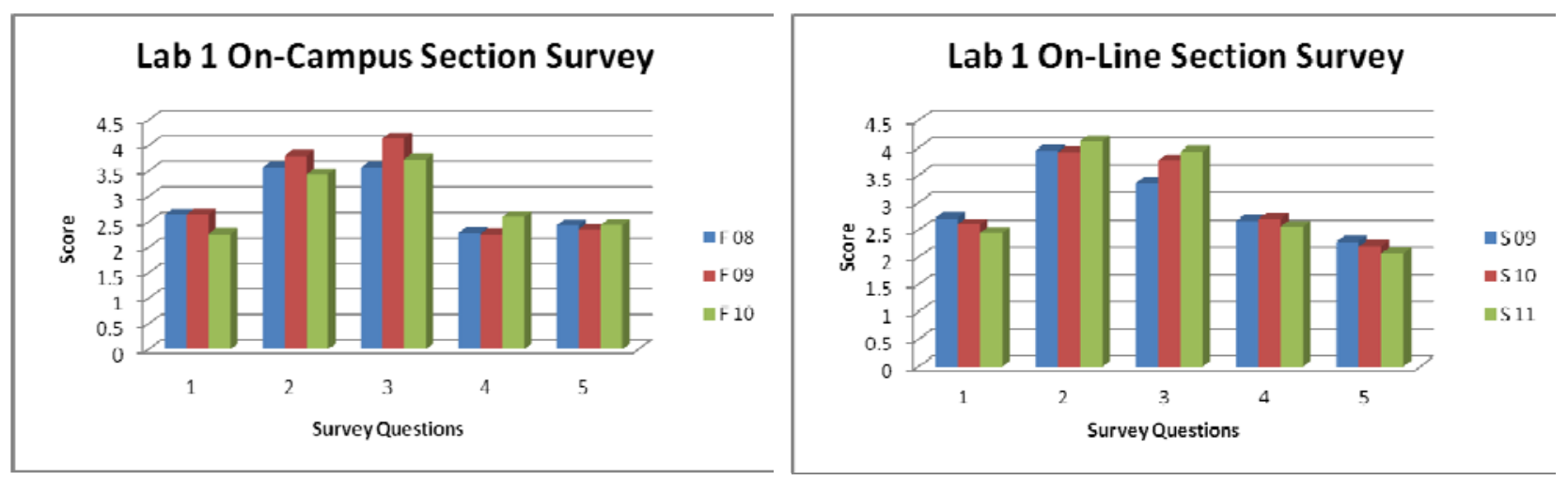

Figure 1. Lab 1 survey and assessment results. 
Table 1(a). Statistical analysis of Lab 1 assessment metric LII.

\begin{tabular}{|l|l|c|c|c|c|}
\hline \multicolumn{7}{|c|}{ Learning Improvement Index (LII) } \\
\hline & & On campus (Fall) & On line (Spring) & t-score & Hypothesis \\
\hline F08- & avg & 0.737 & 0.610 & 0.444 & \multirow{H}{*}{$\boldsymbol{H}_{10}$} \\
\cline { 2 - 4 } S09 & std & 0.985 & 0.523 & & \\
\hline F09- & avg & 0.663 & 0.708 & -0.220 & \multirow{2}{*}{$\boldsymbol{H}_{10}$} \\
\cline { 2 - 4 } S10 & std & 0.721 & 0.742 & & \multirow{2}{*}{$\boldsymbol{H}_{10}$} \\
\hline F10- & avg & 0.728 & 1.188 & -1.418 & \\
\cline { 2 - 4 } S11 & std & 0.769 & 1.169 & & \\
\hline
\end{tabular}

Table 1(b). Statistical analysis of Lab 1 assessment metric NDI.

\begin{tabular}{|l|l|c|c|c|c|}
\hline \multicolumn{7}{|c|}{ Normalized Difficulty Index (NDI) } \\
\hline & & On campus (Fall) & On line (Spring) & t-score & Hypothesis \\
\hline F08- & avg & 0.715 & 0.916 & -1.260 & $\boldsymbol{H}_{10}$ \\
\cline { 2 - 4 } S09 & std & 0.466 & 0.466 & & \multirow{2}{*}{$\boldsymbol{H}_{10}$} \\
\hline F09- & avg & 0.689 & 0.769 & 0.519 & \multirow{2}{*}{$\boldsymbol{H}_{10}$} \\
\cline { 2 - 4 } S10 & std & 0.625 & 0.404 & & \\
\hline F10- & avg & 0.745 & 0.693 & & \\
\cline { 2 - 4 } S11 & std & 0.350 & 0.366 & & \\
\hline
\end{tabular}

Table 1(c). Statistical analysis of Lab 1 assessment metric USI.

\begin{tabular}{|l|l|c|c|c|c|}
\hline \multicolumn{7}{|c|}{ Usability Satisfaction Index (USI) } \\
\hline & & On campus (Fall) & On line (Spring) & t-score & Hypothesis \\
\hline F08- & avg & 0.714 & 0.718 & -0.053 & \multirow{H}{*}{$\boldsymbol{H}_{10}$} \\
\cline { 2 - 4 } S09 & std & 0.232 & 0.192 & & \multirow{2}{*}{$\boldsymbol{H}_{10}$} \\
\hline F09- & avg & 0.705 & 0.760 & -0.836 & \multirow{2}{*}{$\boldsymbol{H}_{10}$} \\
\cline { 2 - 4 } S10 & std & 0.250 & 0.204 & -1.583 & \\
\hline F10- & avg & 0.713 & 0.788 & & \\
\cline { 2 - 4 } S11 & std & 0.146 & 0.154 & & \\
\hline
\end{tabular}

This is a relatively simple lab exercise, and students should be well acquainted with the mathematic knowledge. Therefore this set of results can be served as a benchmark. We observe that the results appear to be relatively stable over the years, and the average scores are in $0.7 \sim$ 0.8 range, which indicates modest learning (LII), difficulty (NDI) and satisfaction (USI) scores. There is no significant difference between the "on campus" and "on line" modes.

\section{Lab2. Projection and Projection Slice Theorem (tomography)}

Student first creates simple 2D objects from isolated points, simple shapes (rectangle, circle, ellipse etc.), and observes their projection (radon) domain presentations. The number and angle of projections are specified by the student. A phantom template is also provided so that student can manipulate the components to created different phantom objects for projection tests. Student 
then use the phantom object to validate projection slice theorem. The process is to take one projection at student specified angle, then display this projection signal, the 1D FFT of this projection, as well as the corresponding slice of the 2D DFT of the phantom image. The student can observe the consistence of these two FFT results at any selected projection angle.
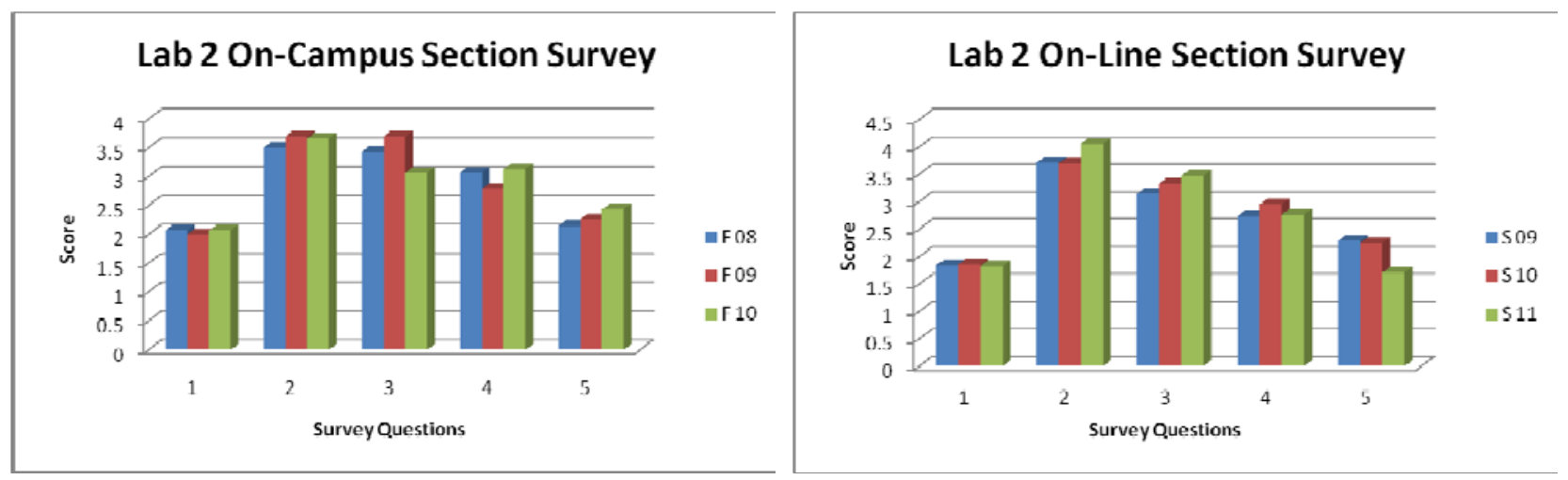

Figure 2. Lab 2 survey and assessment results.

Table 2(a). Statistical analysis of Lab 2 assessment metric LII.

\begin{tabular}{|l|l|c|c|c|c|}
\hline \multicolumn{5}{|c|}{ Learning Improvement Index (LII) } \\
\hline & & On campus (Fall) & On line (Spring) & t-score & Hypothesis \\
\hline F08- & avg & 1.174 & 1.518 & -0.908 & $\boldsymbol{H}_{10}$ \\
\cline { 2 - 4 } S09 & std & 1.082 & 1.147 & & \multirow{2}{*}{$\boldsymbol{H}_{10}$} \\
\hline F09- & avg & 1.367 & 1.425 & -0.177 & \\
\cline { 2 - 4 } S10 & std & 1.154 & 1.117 & -1.556 & $\boldsymbol{H}_{10}$ \\
\hline F10- & avg & 1.231 & 1.897 & & \\
\cline { 2 - 4 } S11 & std & 1.188 & 1.523 & & \\
\hline
\end{tabular}

Table 2(b). Statistical analysis of Lab 2 assessment metric NDI.

\begin{tabular}{|l|l|c|c|c|c|}
\hline \multicolumn{5}{|c|}{ Normalized Difficulty Index (NDI) } \\
\hline & & On campus (Fall) & On line (Spring) & t-score & Hypothesis \\
\hline F08- & avg & 0.952 & 0.987 & -0.257 & $\boldsymbol{H}_{10}$ \\
\cline { 2 - 4 } S09 & std & 0.303 & 0.507 & & \multirow{2}{*}{$\boldsymbol{H}_{10}$} \\
\hline F09- & avg & 0.864 & 0.957 & & \multirow{2}{*}{$\boldsymbol{H}_{10}$} \\
\cline { 2 - 4 } S10 & std & 0.470 & 0.432 & 2.0107 & \\
\hline F10- & avg & 1.072 & 0.853 & & \\
\cline { 2 - 4 } S11 & std & 0.357 & 0.358 & & \\
\hline
\end{tabular}

Table 2(c). Statistical analysis of Lab 2 assessment metric USI.

\begin{tabular}{|l|l|c|c|c|c|}
\hline \multicolumn{5}{|c|}{ Usability Satisfaction Index (USI) } \\
\hline & & On campus (Fall) & On line (Spring) & t-score & Hypothesis \\
\hline F08- & avg & 0.771 & 0.718 & 0.834 & $\boldsymbol{H}_{10}$ \\
\hline
\end{tabular}




\begin{tabular}{|l|l|l|l|l|l|}
\hline S09 & std & 0.190 & 0.182 & & \\
\hline F09- & avg & 0.750 & 0.750 & 0.000 & \multirow{2}{*}{$\boldsymbol{H}_{10}$} \\
\cline { 2 - 4 } S10 & std & 0.128 & 0.194 & & \multirow{2}{*}{$\boldsymbol{H}_{20}$} \\
\hline F10- & avg & 0.713 & 0.860 & & \\
\cline { 2 - 4 } S11 & std & 0.180 & 0.154 & & \\
\hline
\end{tabular}

In this lab students begin to practice the new knowledge they acquired in this course. Compared with Lab 1, a clear increase in learning (LII) and difficulty (NDI) scores are observed, while the satisfaction (USI) scores remain in the range of 07 0.8. Most statistic test results show no difference between on-campus and on-line sections. However one un-equivalent test on USI indicates a higher satisfaction level in on line section (F10-S11).

\section{Lab 3. Frequency domain reconstruction - number of projects, interpolation methods (x- ray $\mathrm{CT}, \mathrm{MRI})$}

Student selects a 2D object and specifies the number of projections, number of samples per projection and projection angles. The projection results are displayed. Each projection is then placed on a 2D frequency domain at corresponding angle, and this process is displayed in both $2 \mathrm{D}$ and $3 \mathrm{D}$ plots. After all projections are placed into this $2 \mathrm{D}$ space, interpolation is performed to create samples at Cartesian grid, and a 2D inverse FFT is performed to generate the reconstruction image. Student is instructed to try a sequence of parameter sets to observe the changes in reconstruction image quality. In particular, frequency domain interpolation can only be observed clearly when number of projections and number of samples per projection are small, but good quality image can only be obtained when these numbers are large. Student will explore these different settings and report the findings.
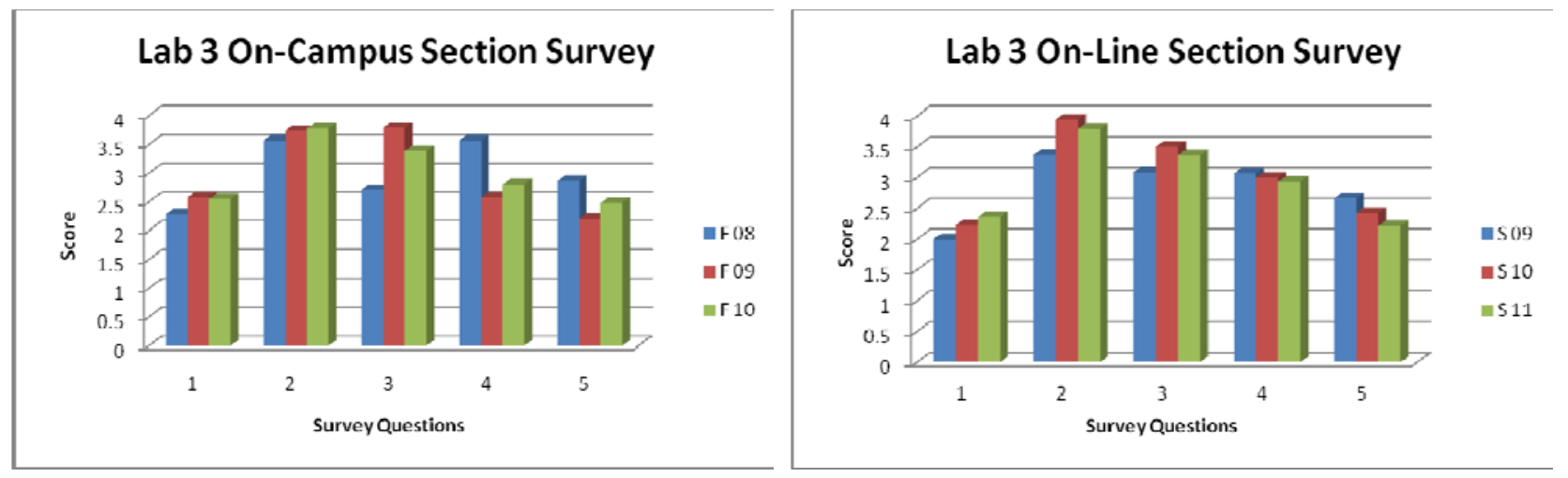

Figure 3. Lab 3 survey and assessment results.

Table 3(a). Statistical analysis of Lab 3 assessment metric LII.

\begin{tabular}{|l|l|c|c|c|c|}
\hline \multicolumn{5}{|c|}{ Learning Improvement Index (LII) } \\
\hline & & On campus (Fall) & On line (Spring) & t-score & Hypothesis \\
\hline F08- & avg & 0.738 & 0.947 & -0.666 & $\boldsymbol{H}_{10}$ \\
\cline { 2 - 6 } S09 & std & 0.666 & 0.876 & & \\
\hline F09- & avg & 0.706 & 1.065 & -1.259 & $\boldsymbol{H}_{10}$ \\
\hline
\end{tabular}




\begin{tabular}{|l|l|l|l|l|l|}
\hline S10 & std & 0.941 & 0.965 & & \\
\hline F10- & avg & 0.809 & 0.887 & -0.271 & \multirow{2}{*}{$\boldsymbol{H}_{10}$} \\
\cline { 2 - 4 } S11 & std & 0.869 & 0.902 & & \\
\hline
\end{tabular}

Table 3(b). Statistical analysis of Lab 3 assessment metric NDI.

\begin{tabular}{|l|l|c|c|c|c|}
\hline \multicolumn{7}{|c|}{ Normalized Difficulty Index (NDI) } \\
\hline & & On campus (Fall) & On line (Spring) & t-score & Hypothesis \\
\hline F08- & avg & 1.405 & 1.388 & 0.048 & \multirow{H}{*}{$\boldsymbol{H}_{10}$} \\
\cline { 2 - 4 } S09 & std & 0.607 & 1.314 & & \multirow{2}{*}{$\boldsymbol{H}_{10}$} \\
\hline F09- & avg & 0.860 & 0.967 & -0.562 & \multirow{2}{*}{$\boldsymbol{H}_{10}$} \\
\cline { 2 - 4 } S10 & std & 0.648 & 0.623 & -0.792 & \\
\hline F10- & avg & 0.859 & 0.979 & & \\
\cline { 2 - 4 } S11 & std & 0.344 & 0.517 & & \\
\hline
\end{tabular}

Table 3(c). Statistical analysis of Lab 3 assessment metric USI.

\begin{tabular}{|l|l|c|c|c|c|}
\hline \multicolumn{7}{|c|}{ Usability Satisfaction Index (USI) } \\
\hline & & On campus (Fall) & On line (Spring) & t-score & Hypothesis \\
\hline F08- & avg & 0.626 & 0.668 & -0.601 & \multirow{H}{*}{$\boldsymbol{H}_{10}$} \\
\cline { 2 - 4 } S09 & std & 0.138 & 0.189 & & \multirow{2}{*}{$\boldsymbol{H}_{10}$} \\
\hline F09- & avg & 0.758 & 0.715 & 0.887 & \multirow{2}{*}{$\boldsymbol{H}_{10}$} \\
\cline { 2 - 4 } S10 & std & 0.171 & 0.149 & -0.927 & \\
\hline F10- & avg & 0.703 & 0.757 & & \\
\cline { 2 - 4 } S11 & std & 0.185 & 0.179 & & \\
\hline
\end{tabular}

Lab 3 becomes more sophisticated and deeper into the new subject. Compared with Lab 2, we see a little reduced learning scores (LII) and the same difficulty scores (NDI). The satisfaction scores (USI) are also a little lower. Again there is no significant difference between two learning modes.

\section{Lab 4. Filtered back projection - number of projections, filters, noise (x-ray CT)}

Student selects a 2D object and specifies a projection angle and number of samples per projection. The 1D projection is displayed. Then student clicks "back-projection", and observes the creation of a 2D back-projection image displayed in both 2D and 3D plots. Student then specifies a series of projection angles, and observed the accumulation of all back-projections into one 2D reconstruction image. Student should see that such reconstruction looks blurred and too bright. Student then selects a filter and applies it to each 1D projection before the backprojection. Student will observe a much clearer reconstruction image from filtered backprojections. Student will further explore different filters, cut-off frequencies of filters, and projections with different levels of induced noise. The filtering effects become more evident. Given the large parameter space, this exercise is rather long and it usually takes students two weeks to complete. 

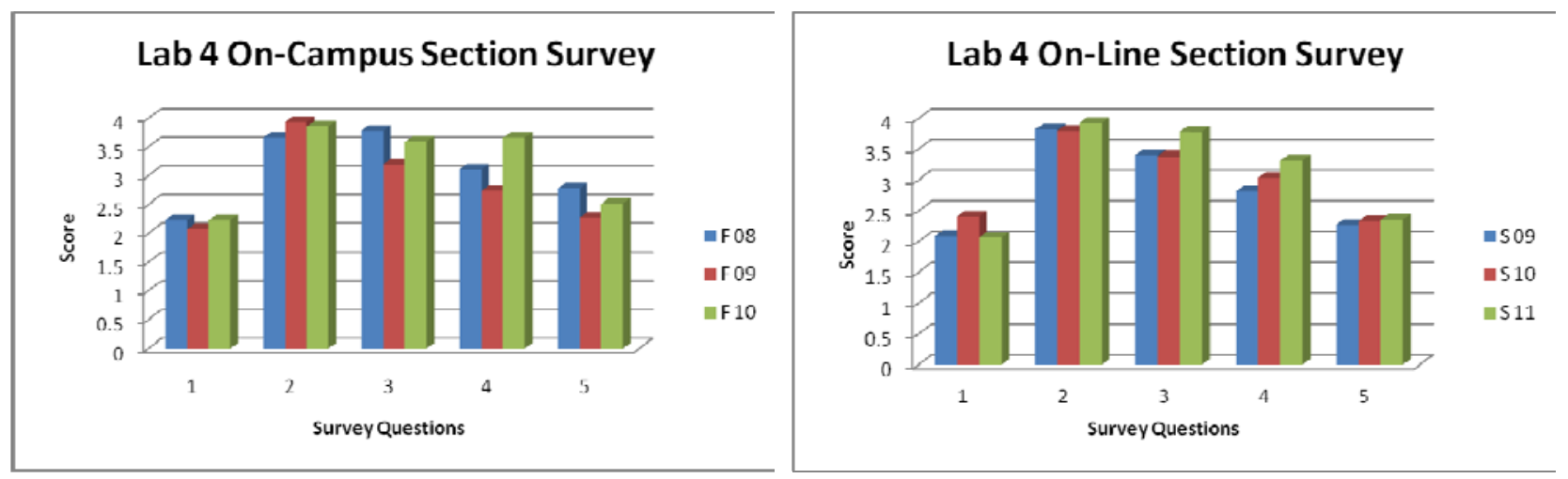

Figure 4. Lab 4 survey and assessment results.

Table 4(a). Statistical analysis of Lab 4 assessment metric LII.

\begin{tabular}{|l|l|c|c|c|c|}
\hline \multicolumn{5}{|c|}{ Learning Improvement Index (LII) } \\
\hline & & On campus (Fall) & On line (Spring) & t-score & Hypothesis \\
\hline F08- & avg & 0.963 & 1.182 & -0.537 & $\boldsymbol{H}_{10}$ \\
\cline { 2 - 4 } S09 & std & 0.992 & 1.116 & & \multirow{2}{*}{$\boldsymbol{H}_{10}$} \\
\hline F09- & avg & 1.222 & 0.796 & & \multirow{2}{*}{$\boldsymbol{H}_{10}$} \\
\cline { 2 - 4 } S10 & std & 1.080 & 0.756 & -0.991 & \\
\hline F10- & avg & 0.966 & 1.304 & & \\
\cline { 2 - 4 } S11 & std & 0.776 & 1.146 & & \\
\hline
\end{tabular}

Table 4(b). Statistical analysis of Lab 4 assessment metric NDI.

\begin{tabular}{|l|l|c|c|c|c|}
\hline \multicolumn{5}{|c|}{ Normalized Difficulty Index (NDI) } \\
\hline & & On campus (Fall) & On line (Spring) & t-score & Hypothesis \\
\hline F08- & avg & 0.904 & 0.918 & -0.080 & $\boldsymbol{H}_{10}$ \\
\cline { 2 - 4 } S09 & std & 0.458 & 0.462 & & \multirow{2}{*}{$\boldsymbol{H}_{10}$} \\
\hline F09- & avg & 1.052 & 1.026 & & \\
\cline { 2 - 4 } S10 & std & 0.721 & 0.647 & \multirow{2}{*}{$\boldsymbol{H}_{10}$} \\
\hline F10- & avg & 1.160 & 0.955 & & \\
\cline { 2 - 4 } S11 & std & 0.840 & 0.425 & & \\
\hline
\end{tabular}

Table 4(c). Statistical analysis of Lab 4 assessment metric USI.

\begin{tabular}{|l|l|c|c|c|c|}
\hline \multicolumn{5}{|c|}{ Usability Satisfaction Index (USI) } \\
\hline & & On campus (Fall) & On line (Spring) & t-score & Hypothesis \\
\hline F08- & avg & 0.644 & 0.745 & -1.692 & $\boldsymbol{H}_{10}$ \\
\cline { 2 - 4 } S09 & std & 0.133 & 0.187 & \multirow{2}{*}{0.203} & $\boldsymbol{H}_{10}$ \\
\hline F09- & avg & 0.747 & 0.733 & & \multirow{2}{*}{$\boldsymbol{H}_{10}$} \\
\cline { 2 - 4 } S10 & std & 0.207 & 0.200 & -0.474 & \\
\hline F10- & avg & 0.696 & 0.729 & & \\
\cline { 2 - 4 } S11 & std & 0.187 & 0.216 & & \\
\hline
\end{tabular}


Lab 4 is an extension to Lab 3, and is more challenging. Compared with Lab 3, we observed slight increase in learning scores (LII), and similar and high difficulty scores (NDI). The satisfaction scores (USI) are about the same. Also, no signification difference between two learning modes is observed.

\section{Lab 5. X-ray attenuation coefficient and survival probability (x-ray)}

Student selects a material from ("adipose", "air", "aluminum", "bone", "copper", "iodine", "lead", "lung", "muscle", "soft tissue", "water"), and changes the incident x-ray energy from 10 to 400 $\mathrm{KeV}$. The mass attenuation coefficient is displayed for each material at each $\mathrm{x}$-ray energy level. Absorption edges for some materials can be observed when the energy increment is small. In the second part, student selects a metal material, an incident x-ray energy and changes the thickness of the material to observe the numbers of survival x-ray photons after the penetration. The results are based on NIST dataset, and there is not much computation involved.
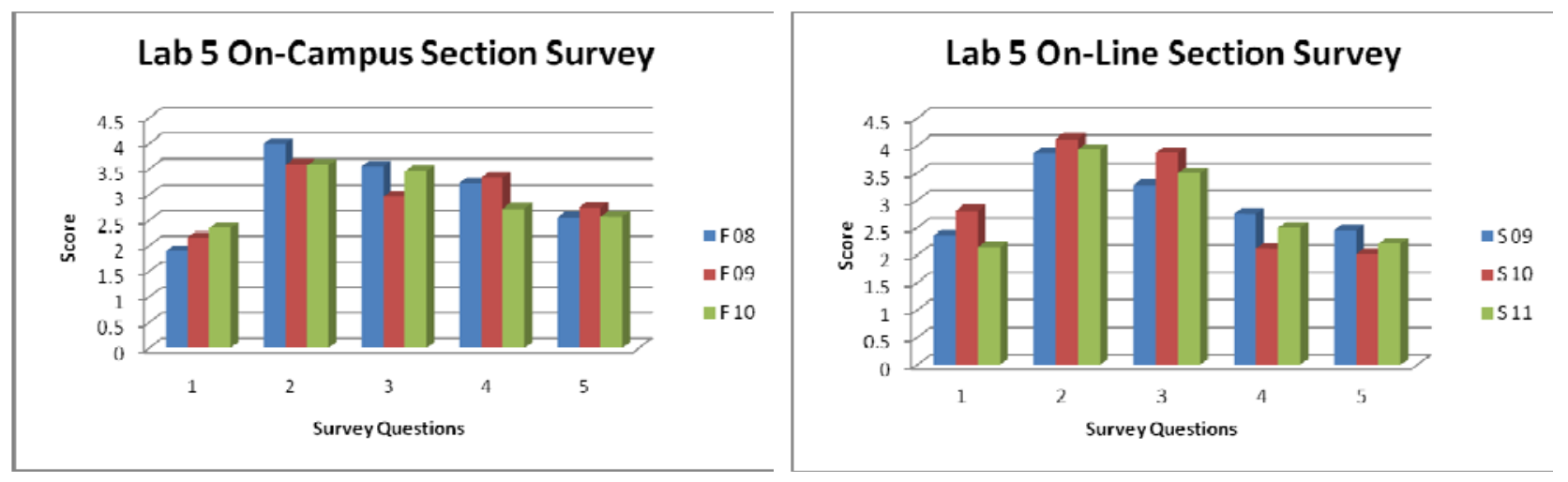

Figure 5. Lab 5 survey and assessment results.

Table 5(a). Statistical analysis of Lab 5 assessment metric LII.

\begin{tabular}{|l|l|c|c|c|c|}
\hline \multicolumn{5}{|c|}{ Learning Improvement Index (LII) } \\
\hline & & On campus (Fall) & On line (Spring) & t-score & Hypothesis \\
\hline F08- & avg & 1.352 & 0.852 & 1.442 & $\boldsymbol{H}_{10}$ \\
\cline { 2 - 4 } S09 & std & 0.868 & 0.873 & & \multirow{2}{*}{$\boldsymbol{H}_{10}$} \\
\hline F09- & avg & 1.056 & 0.678 & & \\
\cline { 2 - 4 } S10 & std & 0.976 & 0.649 & \multirow{2}{*}{$\boldsymbol{H}_{10}$} \\
\hline F10- & avg & 0.883 & 1.095 & & \\
\cline { 2 - 4 } S11 & std & 0.983 & 0.803 & & \\
\hline
\end{tabular}

Table 5(b). Statistical analysis of Lab 5 assessment metric NDI.

\begin{tabular}{|l|l|c|c|c|c|}
\hline \multicolumn{5}{|c|}{ Normalized Difficulty Index (NDI) } \\
\hline & & On campus (Fall) & On line (Spring) & t-score & Hypothesis \\
\hline F08- & avg & 0.954 & 0.946 & 0.038 & $\boldsymbol{H}_{10}$ \\
\cline { 2 - 4 } S09 & std & 0.427 & 0.654 & & \\
\hline F09- & avg & 1.232 & 0.601 & 3.938 & $\boldsymbol{H}_{30}$ \\
\hline
\end{tabular}




\begin{tabular}{|l|l|l|l|l|l|}
\hline S10 & std & 0.480 & 0.394 & & \\
\hline F10- & avg & 0.842 & 0.885 & \multirow{2}{*}{-0.200} & \multirow{2}{*}{$\boldsymbol{H}_{10}$} \\
\cline { 2 - 4 } S11 & std & 0.445 & 0.734 & & \\
\hline
\end{tabular}

Table 5(c). Statistical analysis of Lab 5 assessment metric USI.

\begin{tabular}{|l|l|c|c|c|c|}
\hline \multicolumn{5}{|c|}{ Usability Satisfaction Index (USI) } \\
\hline & & On campus (Fall) & On line (Spring) & t-score & Hypothesis \\
\hline F08- & avg & 0.689 & 0.710 & -0.400 & $\boldsymbol{H}_{10}$ \\
\cline { 2 - 4 } S09 & std & 0.105 & 0.173 & & \multirow{2}{*}{$\boldsymbol{H}_{20}$} \\
\hline F09- & avg & 0.650 & 0.796 & -2.390 & \\
\cline { 2 - 4 } S10 & std & 0.151 & 0.210 & \multirow{2}{*}{-1.042} & $\boldsymbol{H}_{10}$ \\
\hline F10- & avg & 0.685 & 0.757 & & \\
\cline { 2 - 4 } S11 & std & 0.180 & 0.224 & & \\
\hline
\end{tabular}

Lab 5 is quite different from Lab 2 4, and it is more related to physics than mathematics. Also it is relatively simpler. Therefore we observe higher learning scores (LII), and similar difficulty scores (NDI). The satisfaction scores (USI) are lower. Most statistic tests produce equivalent results, except the NDI and USI tests in F09-S10. It appears that on-campus students felt that this lab was more difficult and was less satisfactory. On the other hand, on-line students appeared to feel comfortable with this lab, which is interesting.

\section{Lab 6. NMR signals - precessions, relaxation, basic sequences (MRI)}

Student first gets familiar with 3D vector representation of spin magnetization, by specifying an excitation on the equilibrium vector $\mathrm{Mz}$, and observing the resulting 3D vector. Then student will observe spin dynamics including transverse (T2) relaxation, longitudinal (T1) relaxation, and free precession individually and jointly. Student specifies T1, T2 times, initiates an excitation angle, and then observes the vector changes over time, typically for a range of $1 \sim 2400 \mathrm{~ms}$. The display is progressive for 10 frames per second. At the same time, the student will also observe the FID (free-induced-decay) signal waveform generated from each session. In the second part, student simulates some basic NMR sequences, including saturation recovery (SR) and spin echo (SE). In SR simulation, student specifies the T1, T2 values, an excitation angle, the repetition time (TR), echo time (TE), and repetition number. Student will observe the vector animation and FID that is generated. In SE simulation, student specifies number of spins, e.g. 10, off-resonance frequencies randomly distributed between $-50 \mathrm{~Hz}$ and $50 \mathrm{~Hz}$. Student can observe the animation of all these spin vectors and the aggregated FID signals. In particular, this simulation is very helpful in explaining the divergence and refocus of magnetization on $x-y$ plan in SE. This exercise is also very long, and it usually takes students two weeks to complete. 

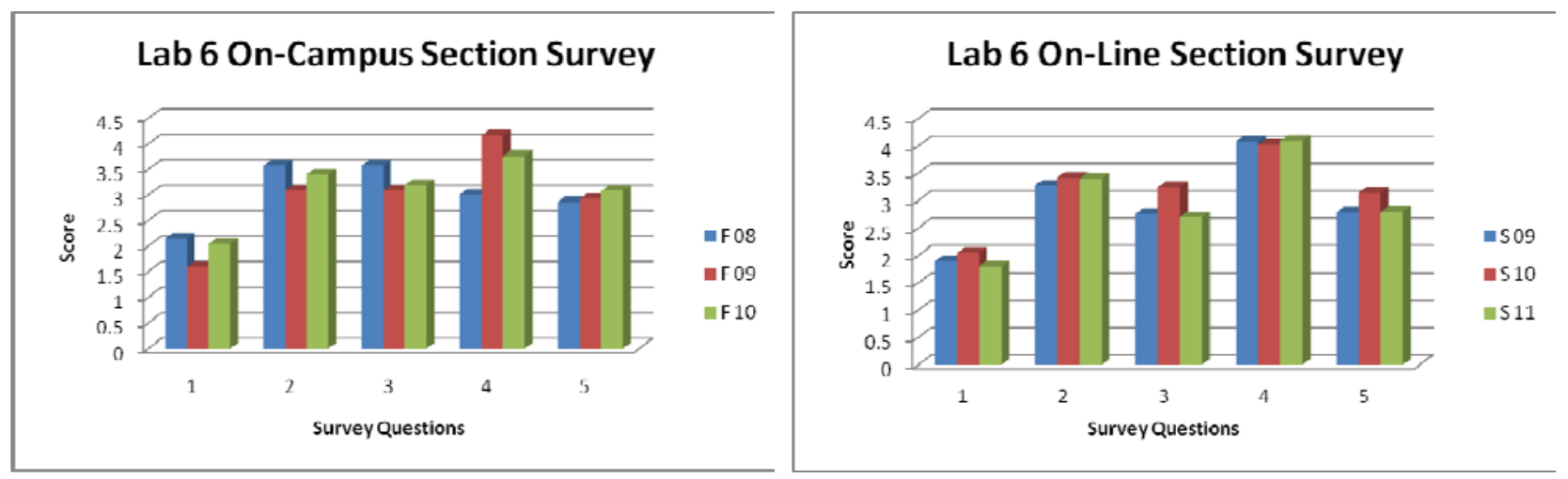

Figure 6. Lab 6 survey and assessment results.

Table 6(a). Statistical analysis of Lab 6 assessment metric LII.

\begin{tabular}{|l|l|c|c|c|c|}
\hline \multicolumn{5}{|c|}{ Learning Improvement Index (LII) } \\
\hline & & On campus (Fall) & On line (Spring) & t-score & Hypothesis \\
\hline F08- & avg & 0.857 & 0.967 & -0.340 & $\boldsymbol{H}_{10}$ \\
\cline { 2 - 4 } S09 & std & 0.690 & 0.905 & & \multirow{2}{*}{$\boldsymbol{H}_{10}$} \\
\hline F09- & avg & 1.153 & 1.001 & & \multirow{2}{*}{$\boldsymbol{H}_{10}$} \\
\cline { 2 - 4 } S10 & std & 0.668 & 0.766 & -0.937 & \\
\hline F10- & avg & 1.050 & 1.358 & & \\
\cline { 2 - 4 } S11 & std & 0.917 & 0.864 & & \\
\hline
\end{tabular}

Table 6(b). Statistical analysis of Lab 6 assessment metric NDI.

\begin{tabular}{|l|l|c|c|c|c|}
\hline \multicolumn{5}{|c|}{ Normalized Difficulty Index (NDI) } \\
\hline & & On campus (Fall) & On line (Spring) & t-score & Hypothesis \\
\hline F08- & avg & 0.948 & 1.763 & -2.614 & $\boldsymbol{H}_{20}$ \\
\cline { 2 - 4 } S09 & std & 0.578 & 1.068 & & \multirow{2}{*}{$\boldsymbol{H}_{10}$} \\
\hline F09- & avg & 1.451 & 1.378 & & \\
\cline { 2 - 4 } S10 & std & 0.515 & 0.602 & -1.041 & $\boldsymbol{H}_{10}$ \\
\hline F10- & avg & 1.334 & 1.583 & & \\
\cline { 2 - 4 } S11 & std & 0.874 & 0.515 & & \\
\hline
\end{tabular}

Table 6(c). Statistical analysis of Lab 6 assessment metric USI.

\begin{tabular}{|l|l|c|c|c|c|}
\hline \multicolumn{5}{|c|}{ Usability Satisfaction Index (USI) } \\
\hline & & On campus (Fall) & On line (Spring) & t-score & Hypothesis \\
\hline F08- & avg & 0.629 & 0.643 & -0.116 & $\boldsymbol{H}_{10}$ \\
\cline { 2 - 4 } S09 & std & 0.315 & 0.233 & \multirow{2}{*}{0.742} & $\boldsymbol{H}_{10}$ \\
\hline F09- & avg & 0.617 & 0.571 & & \multirow{2}{*}{$\boldsymbol{H}_{10}$} \\
\cline { 2 - 4 } S10 & std & 0.159 & 0.203 & -0.972 & \\
\hline F10- & avg & 0.584 & 0.640 & & \\
\cline { 2 - 4 } S11 & std & 0.208 & 0.126 & & \\
\hline
\end{tabular}


Lab 6 is the most challenging lab exercise, because it is mostly mathematical and abstract. We observe high learning scores (LII), high difficulty scores (NDI) and low satisfaction scores (USI). This time, on-line students in F08-S09 had more significant difficult feeling, possibly because of less interaction with the instructor on this very difficult subject.

\section{Lab 7. Brain activation detection in fMRI (image analysis)}

Student is given a functional MRI dataset containing one axial brain slice for 68 time samples. Each image is of 46 by 55 in size. The data was collected by a 1.5T GE Echo Speed Horizon scanner for a finger-tapping test. The paradigm contains 4 on-periods and 5-off periods, which is explained to the student. The first image is displayed, and the student can click any pixel on the image to display the time sequence of that pixel. In the lab instruction, a few active pixels are listed, and student can locate these pixels and see the similarity of these time sequence with the paradigm. Then student is asked to find a few more active pixels, e.g. five. A t-test tool is provided, so student can obtain the t-value for any selected pixel, and can observe that higher tvalues correspond to higher similarity between the selected pixel and the paradigm.
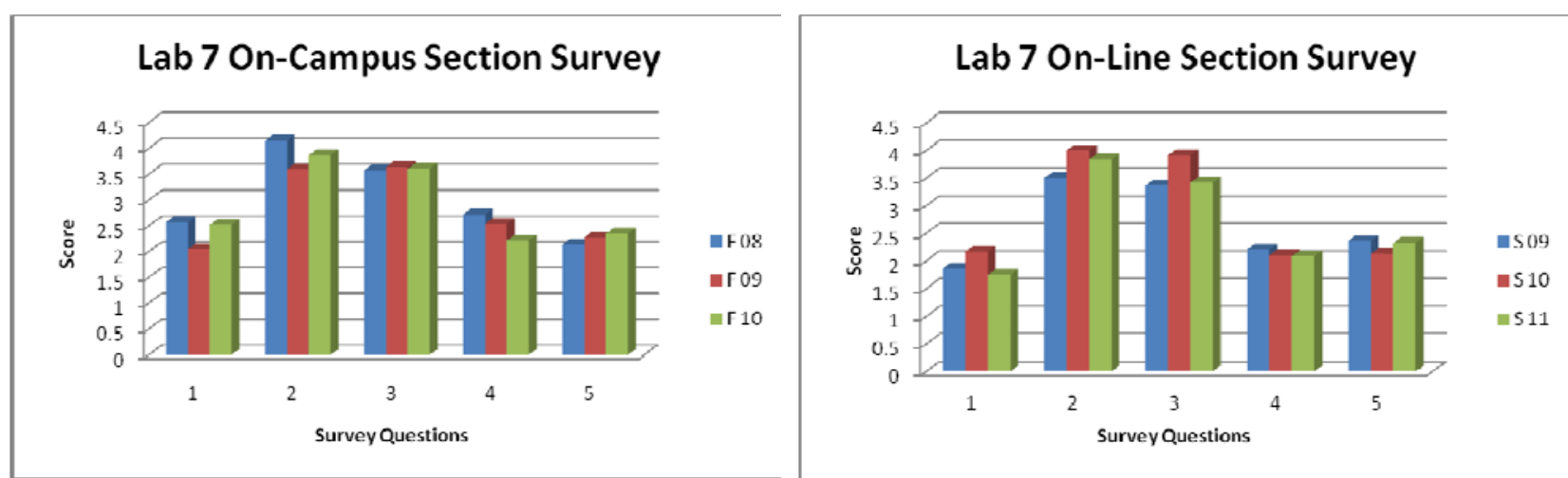

Figure 7. Lab 7 survey and assessment results.

Table 7(a). Statistical analysis of Lab 7 assessment metric LII.

\begin{tabular}{|l|l|c|c|c|c|}
\hline \multicolumn{5}{|c|}{ Learning Improvement Index (LII) } \\
\hline & & On campus (Fall) & On line (Spring) & t-score & Hypothesis \\
\hline F08- & avg & 0.714 & 1.267 & -1.852 & $\boldsymbol{H}_{10}$ \\
\cline { 2 - 4 } S09 & std & 0.405 & 1.115 & & \multirow{2}{*}{$\boldsymbol{H}_{10}$} \\
\hline F09- & avg & 1.067 & 1.247 & -0.447 & \\
\cline { 2 - 4 } S10 & std & 1.087 & 1.145 & & \multirow{2}{*}{$\boldsymbol{H}_{10}$} \\
\hline F10- & avg & 0.841 & 1.556 & & \\
\cline { 2 - 4 } S11 & std & 0.944 & 1.062 & & \\
\hline
\end{tabular}

Table 7(b). Statistical analysis of Lab 7 assessment metric NDI.

\begin{tabular}{|l|l|c|c|c|c|}
\hline \multicolumn{5}{|c|}{ Normalized Difficulty Index (NDI) } \\
\hline & & On campus (Fall) & On line (Spring) & t-score & Hypothesis \\
\hline F08- & avg & 0.800 & 0.714 & 0.507 & $\boldsymbol{H}_{10}$ \\
\cline { 2 - 4 } S09 & std & 0.370 & 0.417 & & \\
\hline
\end{tabular}




\begin{tabular}{|l|l|l|l|l|l|}
\hline F09- & avg & 0.783 & 0.577 & 1.457 & \multirow{2}{*}{$\boldsymbol{H}_{10}$} \\
\cline { 2 - 4 } S10 & std & 0.420 & 0.313 & & \\
\hline F10- & avg & 0.670 & 0.686 & -0.107 & $\boldsymbol{H}_{10}$ \\
\cline { 2 - 4 } S11 & std & 0.316 & 0.460 & & \\
\hline
\end{tabular}

Table 7(c). Statistical analysis of Lab 7 assessment metric USI.

\begin{tabular}{|l|l|c|c|c|c|}
\hline \multicolumn{5}{|c|}{ Usability Satisfaction Index (USI) } & Hypothesis \\
\hline & & On campus (Fall) & On line (Spring) & t-score & \multirow{2}{*}{$\boldsymbol{H}_{10}$} \\
\hline F08- & avg & 0.771 & 0.726 & & \multirow{2}{*}{$\boldsymbol{H}_{10}$} \\
\cline { 2 - 4 } S09 & std & 0.076 & 0.166 & -0.422 & \\
\hline F09- & avg & 0.745 & 0.775 & & \multirow{2}{*}{$\boldsymbol{H}_{10}$} \\
\cline { 2 - 4 } S10 & std & 0.181 & 0.215 & -0.049 & \\
\hline F10- & avg & 0.730 & 0.733 & & \\
\cline { 2 - 4 } S11 & std & 0.187 & 0.156 & & \\
\hline
\end{tabular}

Lab 7 is different from all other lab exercises. It presents an interesting real-world application. Students appeared to enjoy it over the years. We observed high learning scores (LII), and modest difficulty score (NDI) and satisfaction scores (USI). No significant difference between the two learning modes.

\section{Assessment Discussions}

Overall we think the results match our expectation well. We see clearly an increase of score from Question 1 to Question 2 in all of the lab exercises across all sections, which indicates improved understanding of topics under investigation. From Question 3 5 results we see that most of the students seem satisfied with the implementation and usability of the software, although complains of "too much time spent" can be observed from Question 4 results, especially in Lab 3 and 6.

More specifically, from the results we have the following conclusions:

1. Overall the LII scores are quite close between on-campus sections and on-line sections, which suggests that the software labs can provide comparable learning experience to both student groups. This is a significant finding to support such tools for on-line learning.

2. In the first three labs, there is a clear indication that the software is more effective in helping those online students than the regular on-campus students. This may be explained as the fact that online students are more relying on the tools to understand the concepts, while on campus students may depend on instructor in the lectures. The phenomenon appears clear at the beginning of the course, but eventually disappears in later part of the course when the subject becomes more complex and demanding. One possible reason is that, when the course progresses, the fact those online students have less interaction with the instructor is reflected by the increase in their time spent on the exercises and their perceived difficulty levels. 
3. When the difficulty level (NDI) of the lab exercise is high, as seen in Lab 6 (NMR), oncampus students may learn slightly better than the on-line students. Lab 6 is generally considered by students as much more difficult than other labs. Students' behavior in this lab is worth careful study.

4. Lab 7 has relatively low NDIs and high PPIs across all semesters. This may have an interesting implication, i.e. students tend to learn better with simple but application-oriented exercises. In this scenario, on-line students appear to be more satisfied.

In many cases it is very difficult to make conclusive statement given the current survey data. We will continue our studies in the following years. We will also try to introduce direct assessment metrics for each lab exercises. The plan is to design some homework and exam questions, and correlate students' grades with their survey responses. Given our unique advantage of having two sections of the same course, one on campus and one online, we believe that our continuous comparative study will produce helpful findings to the entire online learning community.

\section{Conclusion}

We designed a series of computer lab exercises using SimuRad for an undergraduate medical imaging course, which is regularly offered both on-campus and on-line. Assessments on these labs were obtained through student surveys. We studied the assessment results and obtained some interesting findings. The results generally indicate that this software is a helpful learning tool and its usability is satisfactory. We also observed that students' learning behaviors are slightly different in some instances between the on-campus sections and the on-line sections. We believe that some observations call for further investigations, which may provide insights for developing more effective learning tools, especially for online learning.

\section{Bibliography}

1. A. Webb, Introduction to Biomedical Imaging, IEEE Press Series in Biomedical Engineering, John Wiley \& Sons Inc, 2003.

2. J. T. Bushberg, J. A. Seibert, E. M. Leidholdt, and J. Boone, Essential Physics of Medical Imaging (2nd Ed), Lippincott Williams \& Wilkins, 2002.

3. A. Louie, J. Izatt, and K. Ferrara, "Biomedical Imaging Graduate Education Programs: Imaging Curricula and Imaging Courses", the Whitaker Foundation Biomedical Engineering Education Summit, 2005, http://www.whitaker.org/academic/wrapup.html.

4. C. B. Paschal, "The Need for Effective Biomedical Imaging Education", IEEE Engineering in Medicine and Biology Magazine, Vol. 22, Num. 4, pp. 88-91, July/August 2003.

5. J. E. Greenberg, B. Belgutte, and M. L. Gray, "Hands-On Learning in Biomedical Signal Processing", IEEE Engineering in Medicine and Biology Magazine, Vol. 22, Num. 4, pp. 71-79, July./August 2003.

6. H. Man and A. Ritter, "Computed Tomography in SimuRad - A Medical Imaging Simulation Software", ASEE Annual Conference 2009, Austin TX, Aug. 2009. 